\title{
Computational Auditory Scene Analysis
}

\section{Dan Ellis}

Laboratory for Recognition and Organization of Speech and Audio Dept. Electrical Eng., Columbia Univ., NY USA

dpwe@ee.columbia.edu http://labrosa.ee.columbia.edu/

\section{The Scene Analysis problem ASA and CASA Issues in CASA}




\section{LabROSA Overview}

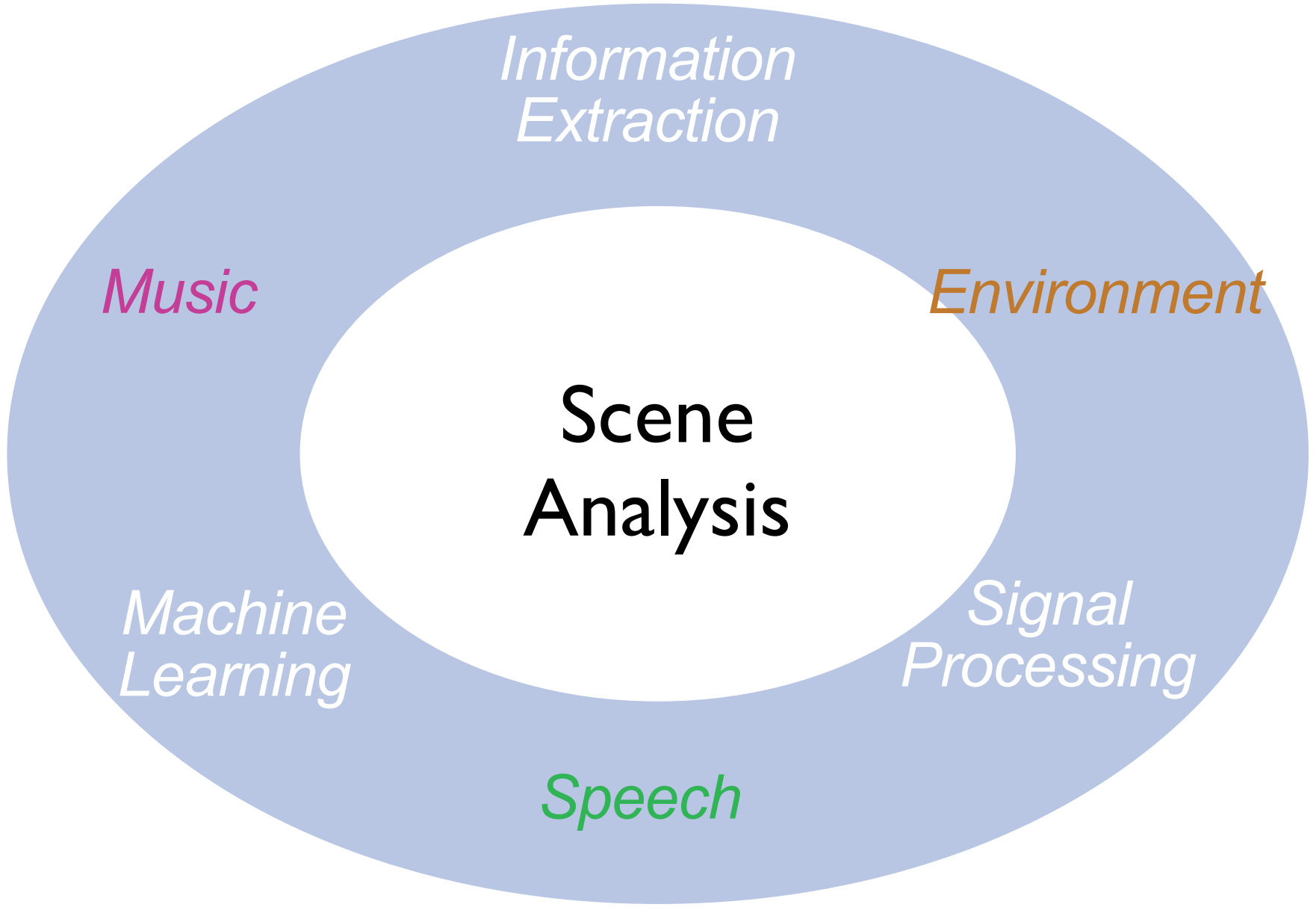

Lab

Comp.Aud. Scene Analysis - Dan Ellis $2005-06-30-2 / 21$ 


\section{Scene Analysis}

- Recover individual sources from scenes ○ .. duplicate the perceptual effect
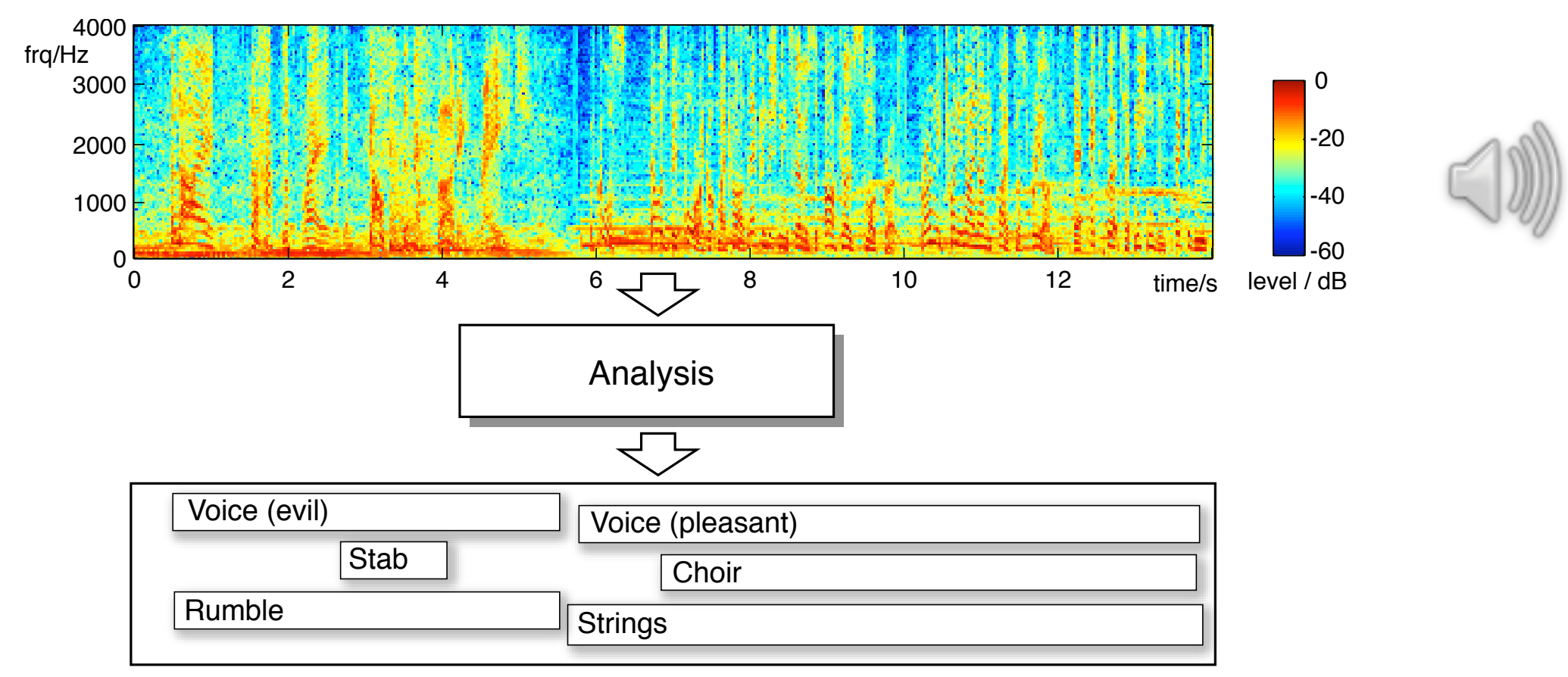

- Problems competing sources, channel effects

- Dimensionality loss

Lab o need additional constraints 


\section{Scene Analysis Systems}

- "Scene Analysis"

o not necessarily separation, recognition, ...

- scene = overlapping objects, ambiguity

\section{- General Framework:}

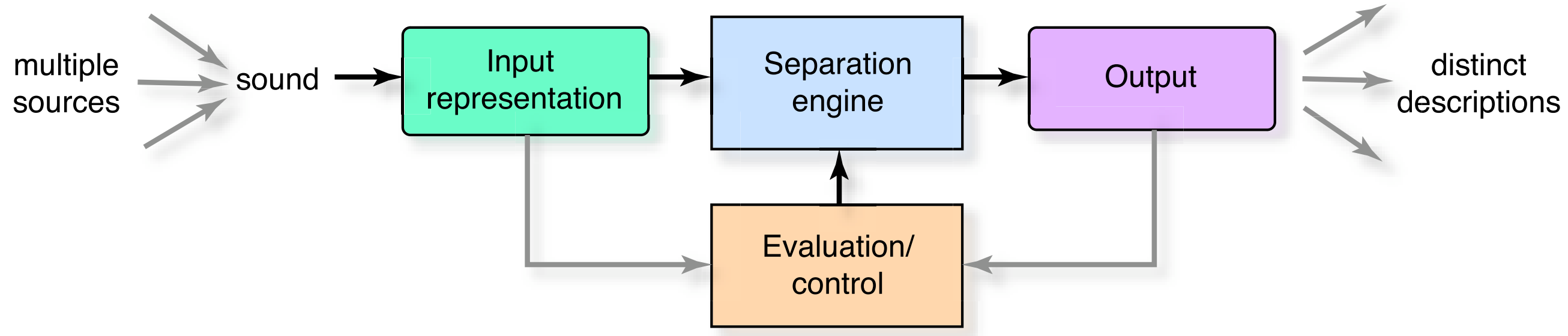

- distinguish input and output representations

o distinguish engine (algorithm) and control (constraints, "computational model") 


\section{Human and Machine Scene Analysis}

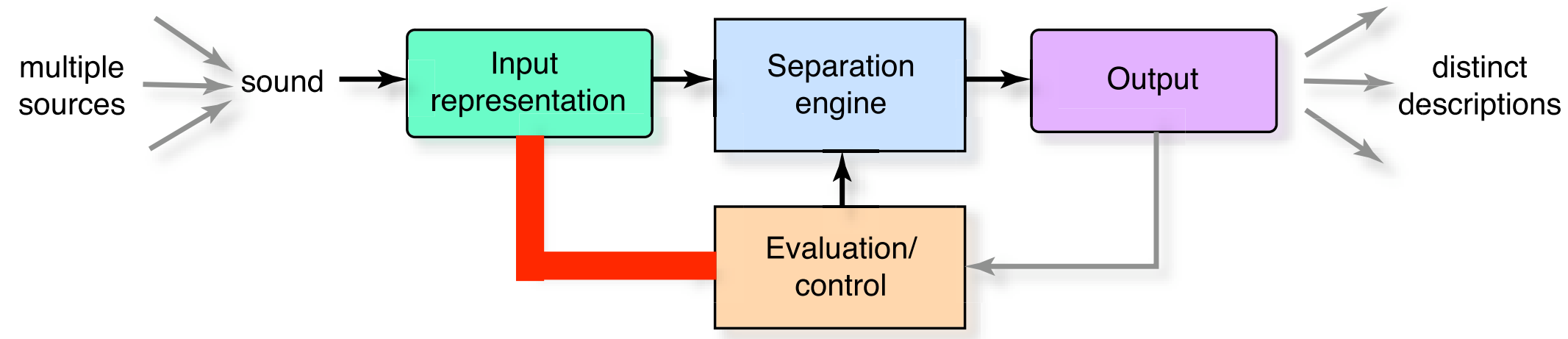

- CASA (Brown'92 et seq.):

- Input: Periodicity, continuity, onset "maps"

- Output: Waveform (or mask)

- Engine:Time-frequency masking

- Control:"Grouping cues" from input

- or: spatial features (Roman, ...) 


\section{Human and Machine Scene Analysis}

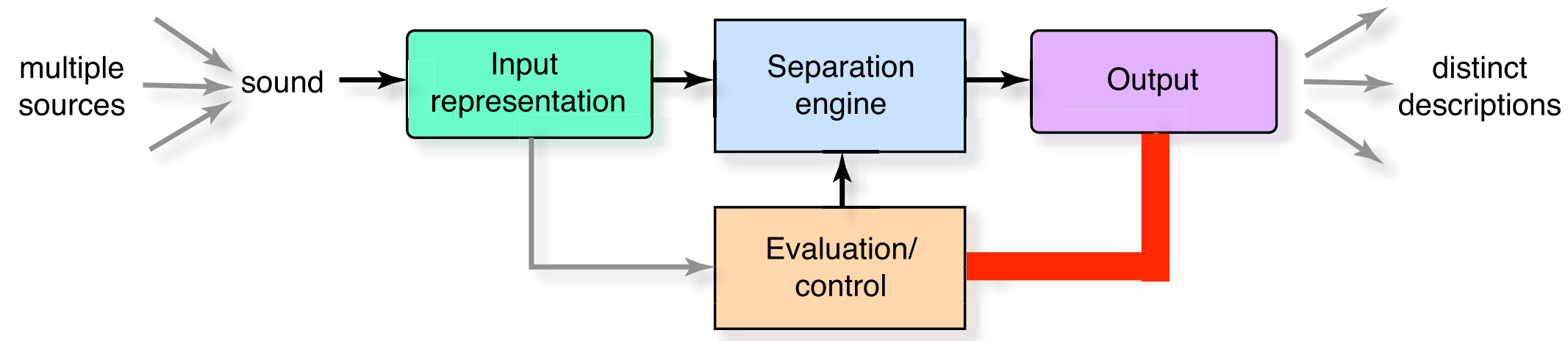

- CASA (e.g. Brown'92):

- ICA (Bell \& Sejnowski et seq.):

- Input: waveform (or STFT)

- Output: waveform (or STFT)

- Engine: cancellation

- Control: statistical independence of outputs

- or energy minimization for beamforming 


\section{Human and Machine Scene Analysis}

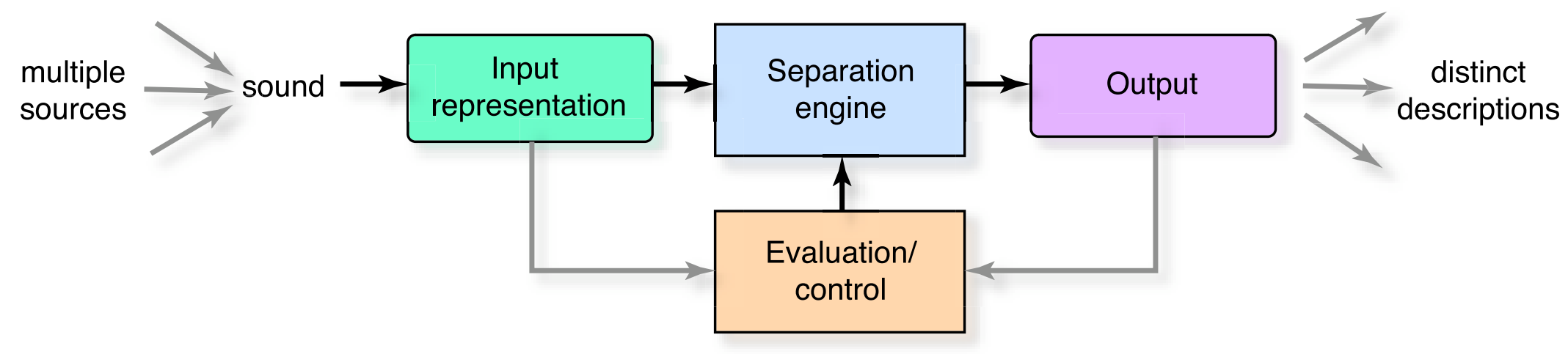

- CASA (e.g. Brown'92):

- ICA (Bell \& Sejnowski et seq.):

- Human Listeners:

- Input: excitation patterns ...

- Output: percepts ...

o Engine:?

- Control: find a plausible explanation 


\section{Auditory Scene Analysis}

(Bregman 1990)

- How do people analyze sound mixtures?

o break mixture into small elements (in time-freq)

- elements are grouped in to sources using cues

- sources have aggregate attributes

- Grouping rules (Darwin, Carlyon, ...):

- cues: common onset/offset/modulation, harmonicity, spatial location, ...

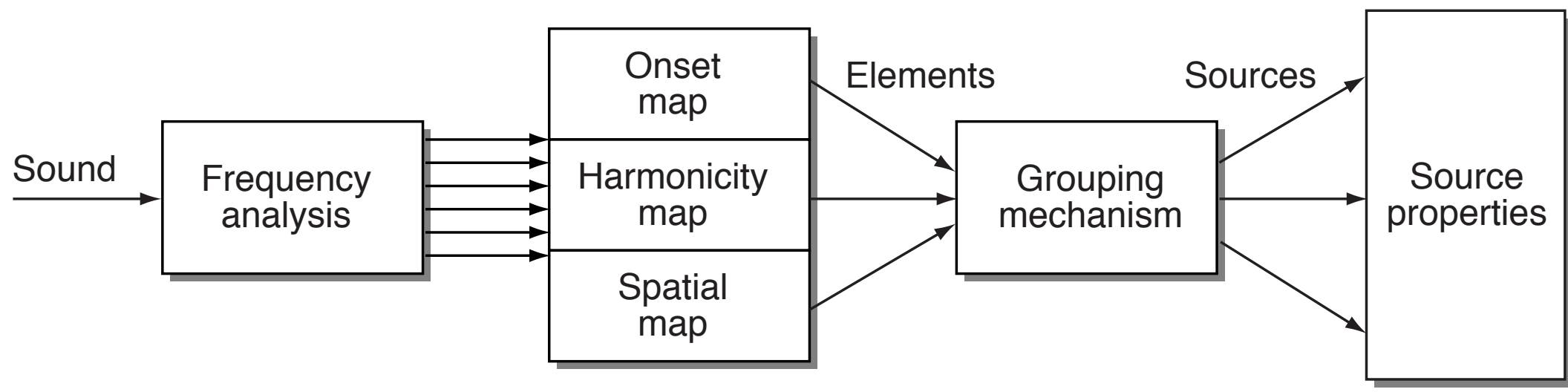

Lab

Comp.Aud. Scene Analysis - Dan Ellis

(after Darwin 1996)

2005-06-30 - 8/21 


\section{Grouping cues}

- Main cues: Harmonicity + Onset

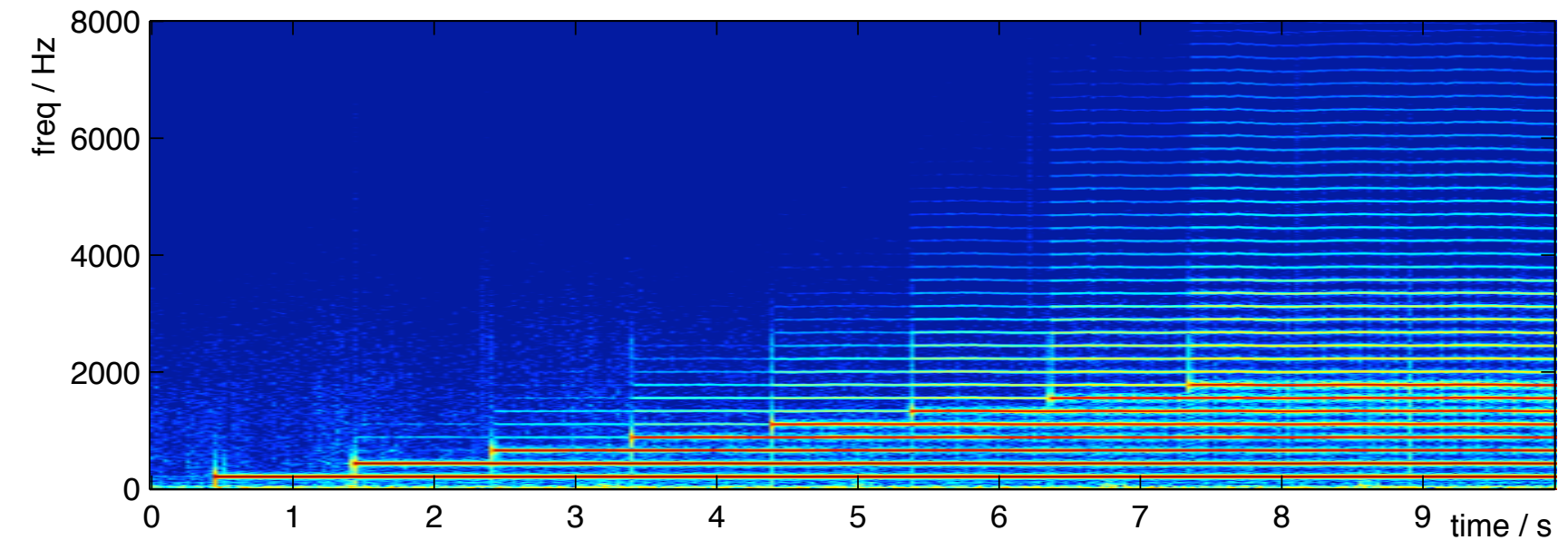

o not necessarily consistent!

- Other cues:

- spatial information

- 'schema' - learned patterns

- Cues $\approx$ constraints 


\section{Bottom-up CASA}

(Brown'92, Hu \& Wang'02)

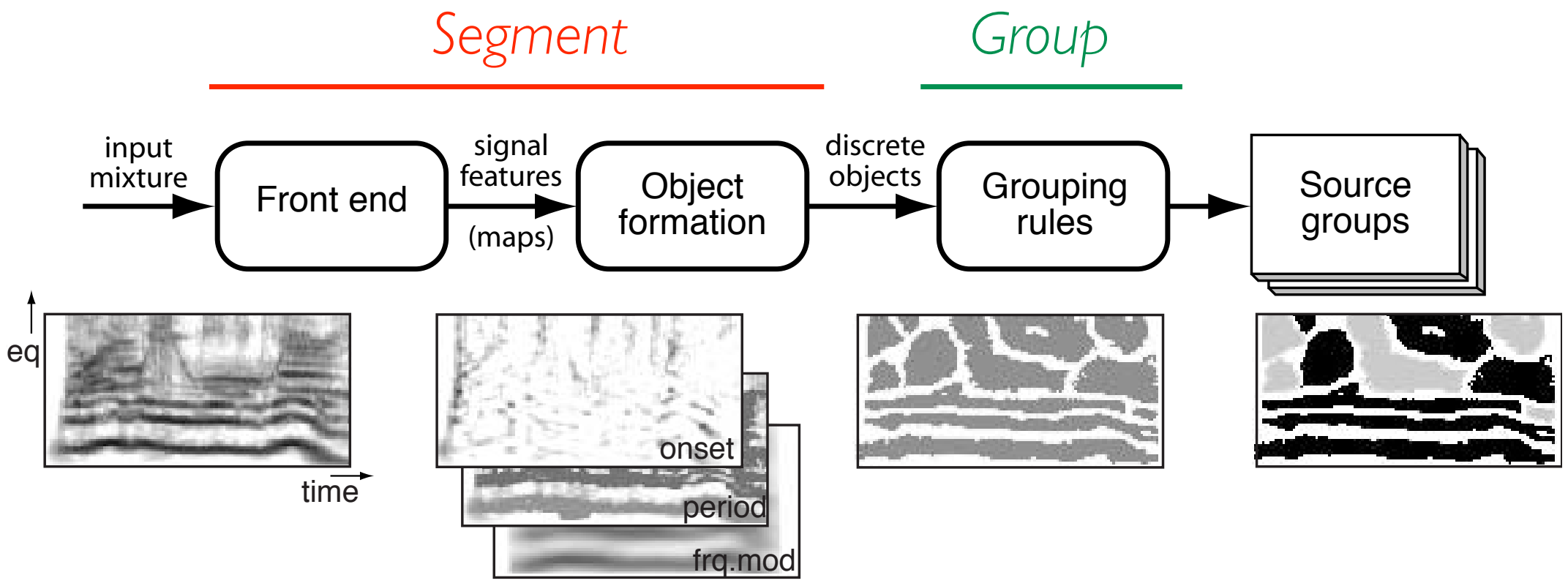

- Literal implementation of psychoacoustics

o segment time-frequency into elements

- group into sources

- Output via time-frequency masking

Lab

o i.e. time-varying filter

Comp.Aud. Scene Analysis - Dan Ellis

$2005-06-30-|0 / 2|$ 


\section{Correlogram front-end (Slaney'90 et seq.)}

- Periodic modulation as 3rd separating axis

- envelope to handle unresolved harmonics

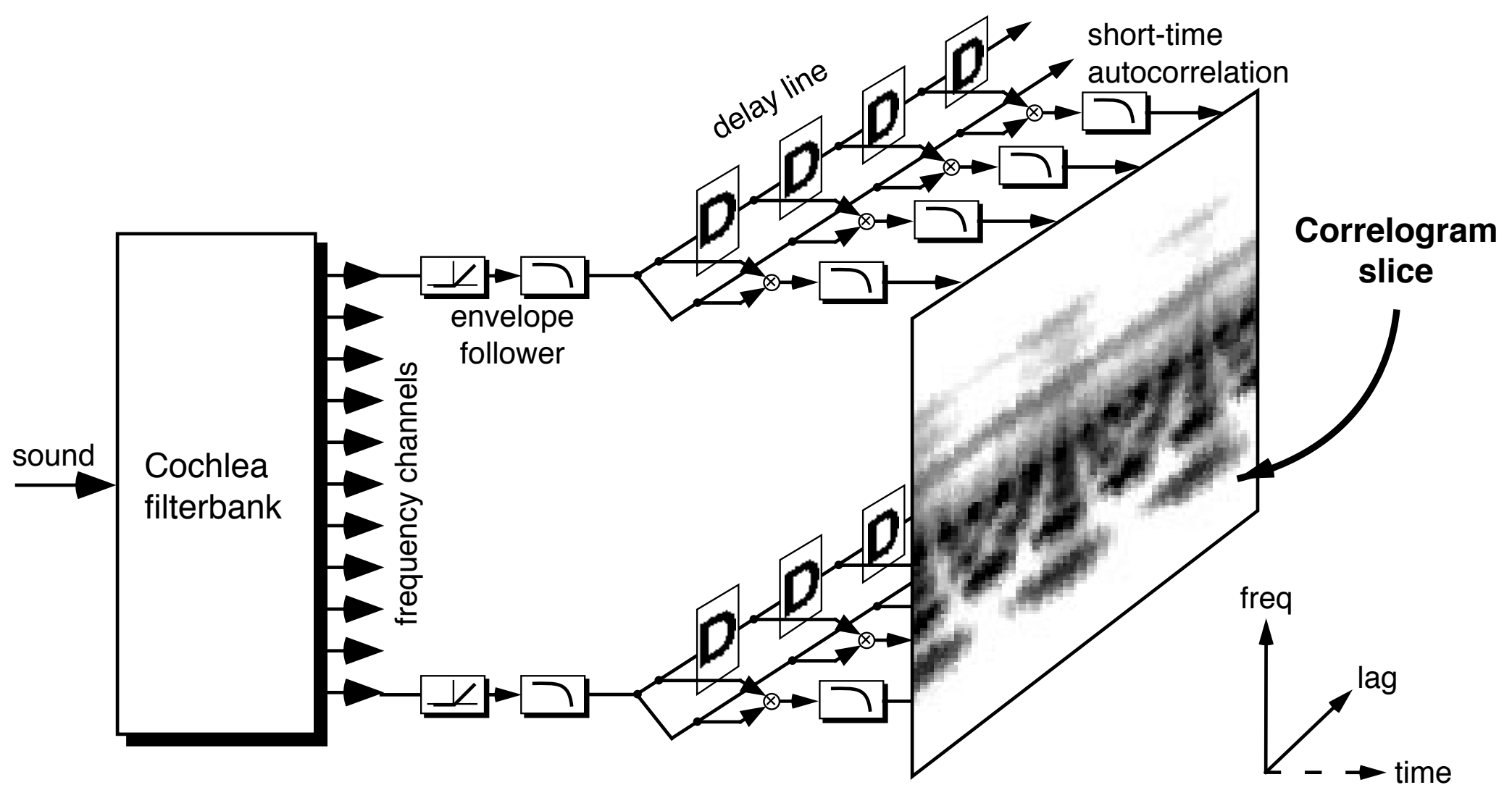

Lab

Comp.Aud. Scene Analysis - Dan Ellis $2005-06-30-|| / 2 \mid$ 


\section{"Weft" Periodic Elements}

(Ellis'96)

\section{- Represent harmonics without grouping?}

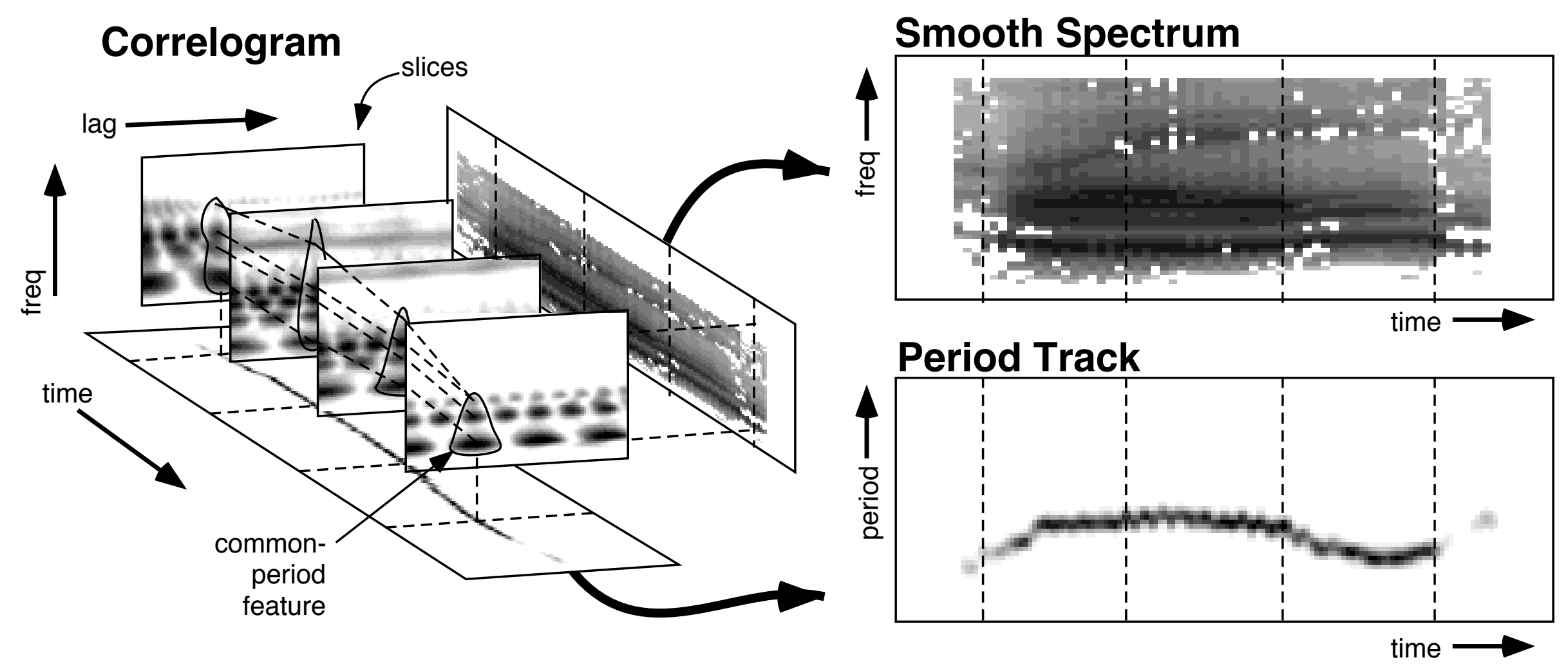

- hard to separate multiple pitch tracks

Lab

Comp.Aud. Scene Analysis - Dan Ellis $2005-06-30-|2 / 2|$ 


\section{CASA Output}

- Time-Frequency masked reconstruction
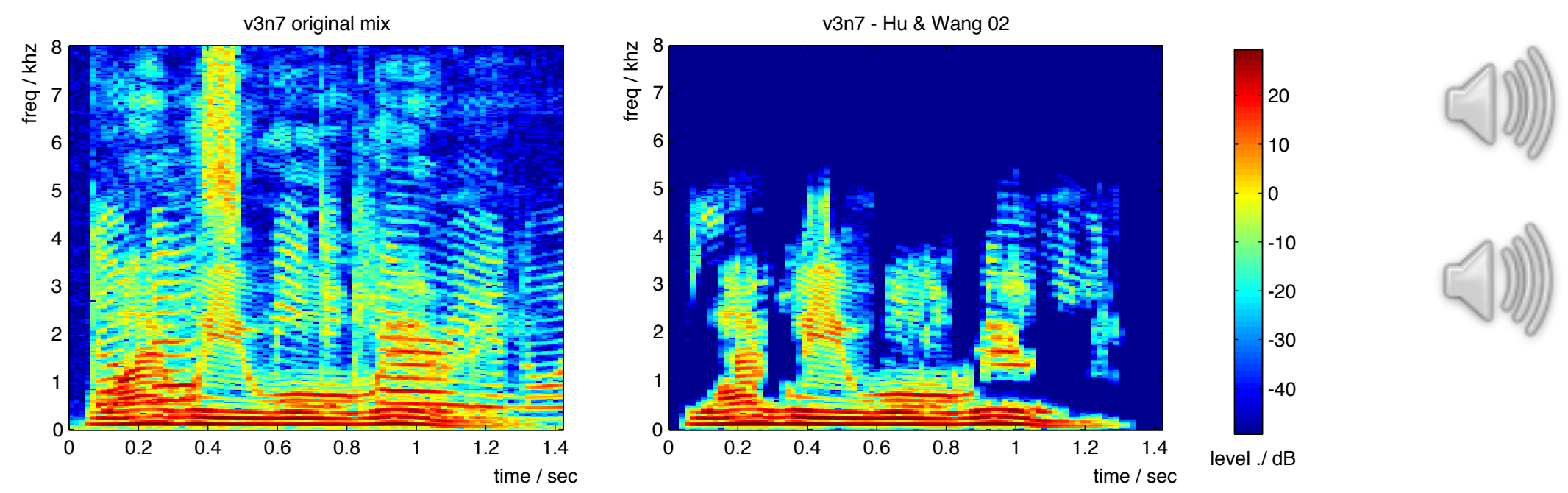

o works surprisingly well (for speech?)

o cannot undo overlapping energy $(<20 \%$ ?)

- applicable to reverberation also?

- Or: parametric resynthesis

o e.g. 'wefts', speech synthesizer 


\section{Challenges for CASA}

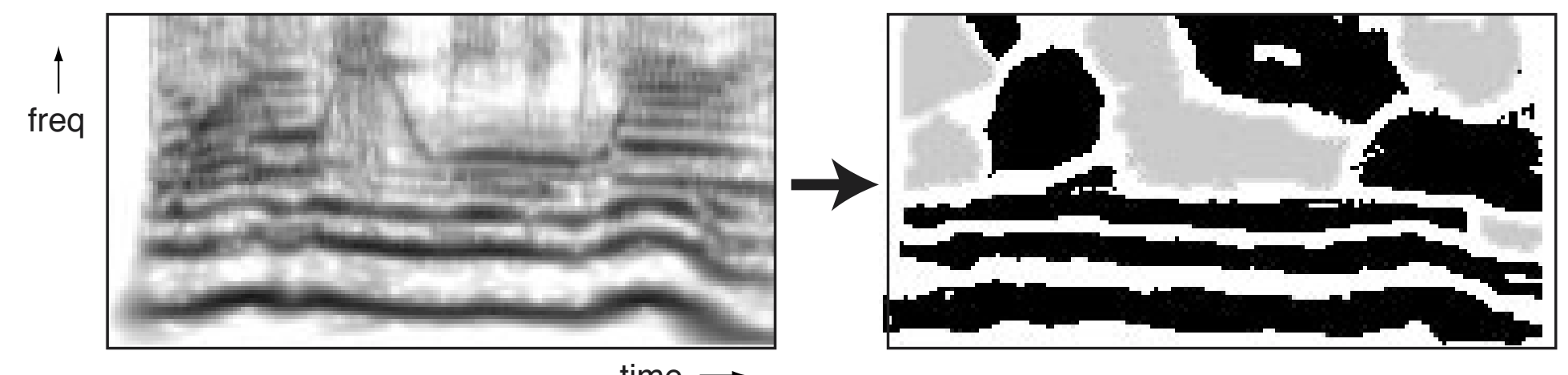

- Circumscribing time-frequency elements

o need to have 'regions', but hard to find

- Periodicity is the primary cue

o how to handle aperiodic energy?

- Bottom-up leaves no ambiguity or context - how to model illusions/interpolations?

- Need to group over longer timespans - local properties not enough 


\section{Model-based integration}

- How to represent high-level constraints? How to integrate disparate fragments?

- "Speech fragment decoder" (Barker et al. '05)

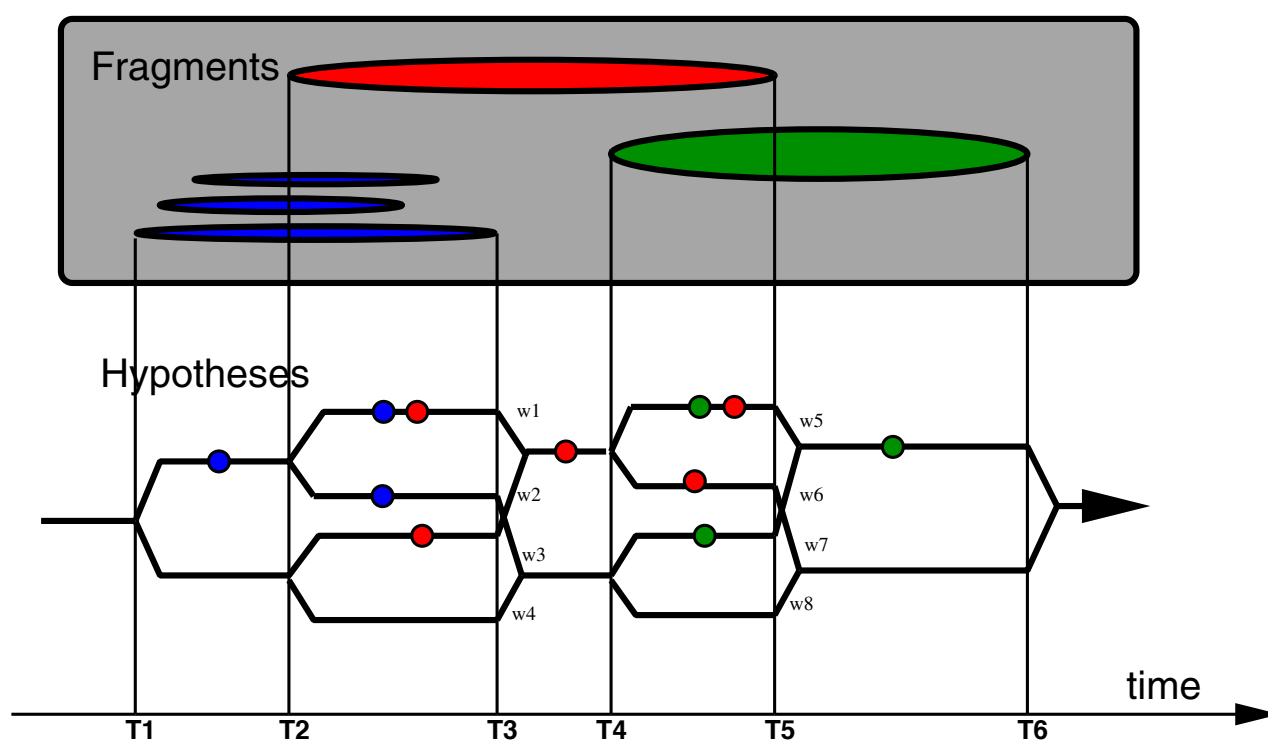

- model of source (e.g. speech recognition HMM) to say which parts go together 


\section{Disambiguation}

- Scene $\Rightarrow$ multiple possible explanations Analysis $\Rightarrow$ choose most reasonable one

- Most reasonable means...
- consistent with grouping cues (CASA)
- independent sources (ICA)
o consistent with experience ... (human)
$\circ \max P\left(\left\{S_{i}\right\} \mid X\right) \propto P\left(X \mid\left\{S_{i}\right\}\right) P\left(\left\{S_{i}\right\}\right)$

- i.e. some kind of constraints to disambiguate - Learning as the source of this disambiguation knowledge 


\section{Recognition for Separation}

- Speech recognizers embody knowledge

- trained on 100s of hours of speech

- use them as a 'reasonableness' measure

- e.g. Seltzer, Raj, Reyes:
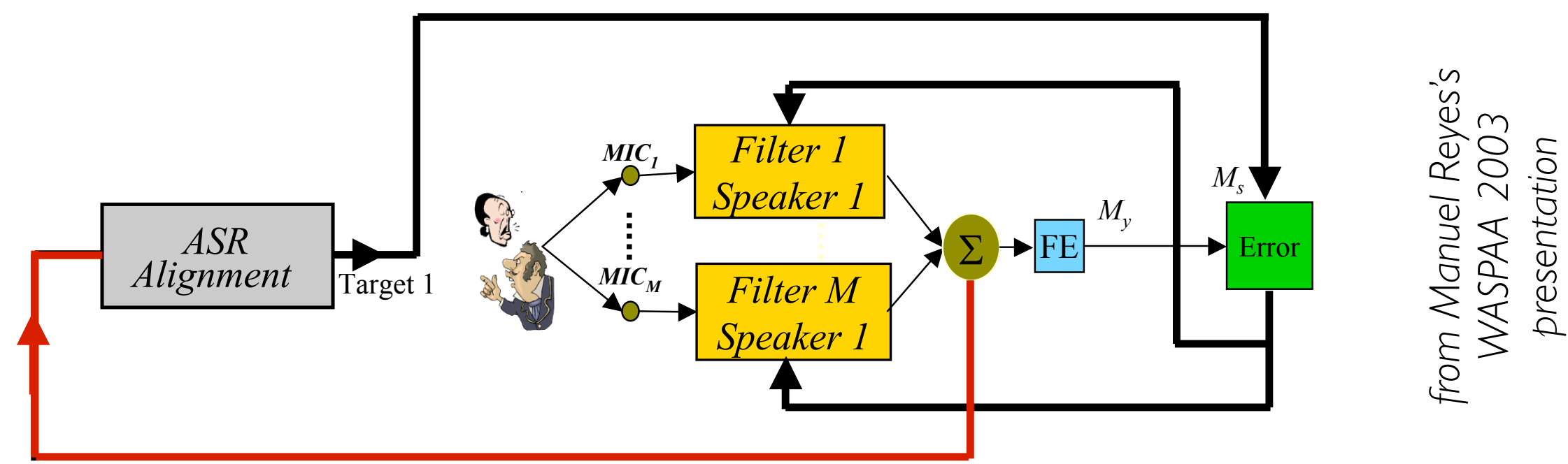

- speech recognizer's best-match provides optimization target 


\section{Obliteration and Outputs}

- Perfect separation is rarely possible

- e.g. cancellation after psychoacoustic coding?

o strong interference will obliterate part of target

- What should the output be?

- can fill-in missing-data holes using source models

- 'pretend' we observed the full signal

- but: hides observed/inferred distinction

- output internal model state instead?

- e.g. ASR output

- synthesize with "minimally informative noise" 


\section{Practical CASA?}

- When will CASA be useful?

o no agreed way to measure progress!

o intelligibility is a novel idea

- Obstacles:

- graceful degradation

- effect of distortions

- unpitched sounds

- computation

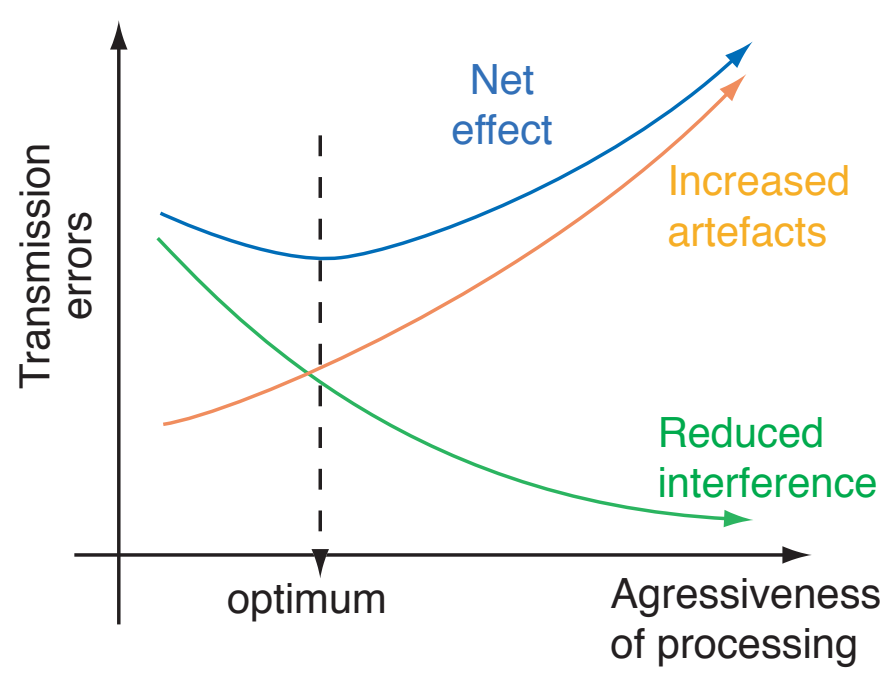

- look-ahead

o integration with multichannel techniques

- sequential or all-at-once? 


\section{Current CASA work}

- Handling unvoiced events (OSU)

- Partial recognition \& grouping (Sheffield)

- Model-based separation (Columbia)

- Spatial cues?

- Dereverberation? 


\section{Conclusions}

- Framework for scene analysis

- Input, Output, Engine, Control

- Auditory Scene Analysis

o in humans and machines

- Scene analysis as Disambiguation

- finding the additional constraints

- Big problems still to overcome 